\title{
FACT AND THEORY IN ELEMENTARY CHEMISTRY.
}

\author{
By IRA D. GARARD, \\ High School, Grove City, Pa.
}

Every teacher of elementary chemistry is confronted with the problem of presenting to the student the simple facts of the science in a logical and systematic way. Hè must explain the relationship of isolated facts in such a manner that the student may be able to generalize for himself and not be dependent on memory for effects and results, for the encyclopedic method is neither easy nor scientific.

Many difficulties are met with and the subject becomes dry and uninteresting. There are always those students who "never get anything" and then there are those who "get it in spite of the teacher"; but those with whom we are most concerned are the majority who take study as a matter of course and do not attack problems with persistency. These are the students that must be assisted by the instruction of the teacher.

As we look back to the time when we finished our elementary chemistry, we likely recall a hazy. jumble of fact and fiction. Atoms seemed as real as metals and chemical action as theoretical as the ether. In fact if we retained any clear conception of chemical fact it was the odor of hydrogen sulphide, the nauseating fumes of nitrogen peroxide, the explosion of an oxyhydrogen mixture, or something else new enough and pronounced enough to impress the senses. Together with these of course, we recall the uses of a few elements or compounds and possibly the explanation of a few everyday phenomena, but we had no clear idea of either the important facts of the science or its importance.

The reason for this condition of students who have completed a year of chemistry seems to me to lie in the failure of text and teacher to keep fact and theory, or hypothesis, separated. It is not uncommon to find ourselves saying that two atoms of hydrogen combine with one of oxygen to form one molecule of water. Again we find ourselves saying that copper sulphate becomes a white powder and loses a certain weight when it is heated because five molecules of water are corrbined in each molecule of the crystal and these are driven off by the heat. On the other hand we say that if a definite amount of any compound be decomposed, it always yields a definite amount of each element that composes it. In the former case, we were talking theory and in the latter 
fact, but we used the same sort of expression in both cases. This gives the student the impression that molecules and atoms of substances are as real as grams or liters of that substance and no amount of drill on the atomic theory as such will serve to disillusionize him.

It seems to me that the remedy for this condition lies in the teaching of fact first and hypothesis later. The student must have the fact and clearly understand it, before he can grasp any explanation of the cause of that fact. To present the atomic theory does not accomplish any definite result. The student may understand its provisions, but he will not see any use for such a theory, unless he first has clearly in mind all of the facts which it endeavors to explain. Some of the more modern textbooks are working toward that end, but most of them are very conservative. The earlier texts presented the atomic theory first and then fitted the subsequent facts into it. Some follow that plan still. Many of them treat oxygen, hydrogen, and possibly chlorine and then the atomic theory just preceding the chemical calculations. This is done to establish a basis for chemical calculations we are told. That is, the student must understand atomic and molecular weight in order to calculate the proportions involved in a reaction. True enough, but let us analyze the situation. We define atomic weight as, "the number which expresses how many times its atom is as heavy as the hydrogen atom," or in some similar terms. Molecular weight, we may define in terms of the oxygen or hydrogen standard or simply call it the sum of the weights of the atoms in the molectules. In either case, the definition is hypothetical and the high school student will ask, "How do you weigh an atom?". This question is almost inevitable from the novice who is always impressed by the description of an atom and the attempt to show how small it must be. Then we go through the process carefully and explain that we do not weigh an atom but that the atomic weight is only a determination of relative weights calculated from vapor densities and the acceptance of Avagadro's hypothesis. This latter assumption is seldom clear to an elementary student and as a result he does not obtain a clear idea of atomic and molecular weights, or if he does he considers them merely theoretical and consequently of little importance. The laboratory work may serve to clear the subject up somewhat, but usually not as well as might be desired for the student cannot associate gram quantities with theoretical numbers.

It seems to me that the most logical course to follow is, as I 
stated above, the presentation of fact first. Oxygen and hydrogen may be studied as is customary, but when quantities or compositions are introduced, treat them as the facts which they are. If water be decomposed, nine grams produce eight of oxygen and one of hydrogen; hydrochloric acid produces 1 gram of hydrogen and 35.5 grams of chlorine, etc. These facts are easily grasped by the students. I might say, however, that a danger exists in the unfortunate confusion of the terms-combining weight, reacting weight, equivalent weight, and atomic weightand some definite meaning for these terms must be followed, preferably the meaning given in the text which is used by the student.

The next step is to generalize from these specific cases of relative weights. Molecular and atomic weights may be introduced, but they must be defined from a fact basis, i. e., one that may be used for a laboratory determination. Some of our physical chemists have defined molecular weight as the weight of 22.4 liters of a gaseous compound under standard conditions, or that weight which will produce an osmotic pressure of 22.4 atmospheres when in one liter of solution. The question of why the number 22.4 was selected, will arise, but may be dismissed as arbitrary except for a historical reason which may be given when the atomic theory is presented later in the course, and even when this is given, it obviously remains arbitrary.

Some crude quantitative vapor density determinations should be made before the class to drive home the idea of actual weights and convey some idea of laboratory methods. The demonstrations of osmotic pressure do not need to be quantitative, but they should show what is meant by osmotic pressure and that it varies with the concentration of the solutions. The student will easily see that the amount of the pressure could be measured.

If we define molecular weight as suggested above, then we may designate the atomic weight as the smallest combining weight of an element ever found in any compound of that element. The method of its calculation may easily be shown, i. e., find the molecular weight of the compound, analyze it for the percentage of constituent elements, and then multiply the former by these percentages. That this gives the combining weights of the elements involved, is obvious. After several examples have been given, we may point out that the smallest combining weight of each element ever found is taken as its atomic weight. This may also be expressed in grams and is still fact. 
The terms "atomic weight" and "molecular weight" are not good, but may be explained as of historical origin and having nothing to do with atoms or molecules.

Symbols, formulae, and equations may be used and explained without the aid of any theory. For example $\mathrm{H}_{2} \mathrm{O}$ represents one molecular weight of water consisting of two atomic weights of hydrogen and one of oxygen, i. e., 2 grams of hydrogen to every 16 grams of oxygen. Also $\mathrm{C}_{2} \mathrm{H}_{6} \mathrm{O}$ represents one molecular weight of alcohol containing two atomic weights of carbon, six of hydrogen, and one of oxygen. Further $\mathrm{O}_{2}$ is so written because one molecular weight of free oxygen is found to contain two atomic weights, i. e., 22.4 liters weigh 32 grams, while the atomic weight is 16 grams.

Reactions may be similarly treated. For example,

$$
\mathrm{CaCO}_{3} \rightarrow \mathrm{CaO}+\mathrm{CO}_{2}
$$

simply means that one molecular weight (100 grams) of calcium carbonate, under certain conditions, breaks down and forms one molecular weight (56 grams) of lime and one (44 grams) of carbon dioxide which latter has a volume of 22.4 liters, since it is a gas.

Reactions involving relative gas volumes also submit to this treatment and valency is simplified. We may say that elements have different powers of combining with each other, e. g., one atomic weight of oxygen is found combined with two of hydrogen while one of chlorine unites with only one of hydrogen. So we say that oxygen has a combining power or valence of two, and chlorine has a combining power or valence of one, since hydrogen has been chosen as the arbitrary standard. All calculations may be performed and facts stated in these demonstrable terms as well as in theoretical ones, and the student is not confused as to fact and theory because he has had none of the latter. After he has been drilled in calculations and performed a few quantitative experiments, then we may say that there have been many hypotheses advanced to explain why these facts are true and teach the atomic theory as the best accepted one. It should be emphasized that while all the facts support this theory, it cannot be proved; but the facts and laws are unalterable and cannot be affected by the proof or disproof of any theory. 